\title{
Respuesta de variedades de maíz (Zea mays L.) a diferentes fuentes de fertilización en el Valle de Puebla
}

\section{Response of maize genotypes (Zea mays L.) to different fertilizers sources in the Valley of Puebla}

\author{
Rafael Alvarado Teyssier ${ }^{1}$, Ernesto Aceves Ruiz ${ }^{2 \ddagger}$, Juan de Dios Guerrero Rodríguez², \\ José Isabel Olvera Hernández ${ }^{2}$, Ángel Bustamante González², \\ Samuel Vargas López ${ }^{2}$ y José Hilario Hernández Salgado²
}

\footnotetext{
${ }^{1}$ Servicios Integrales de Capacitación, Educación y Consultoria Agropecuaria e Industrial S. C. Av. Francisco I. Madero No. 8 Col. Moyotzingo. 74000 San Martín Texmelucan, Puebla, México.

${ }^{2}$ Colegio de Postgraduados, Campus Puebla. Carretera federal México-Puebla km 125.5. 72130 Puebla, Puebla, México.

¥ Autor responsable (ruiz@colpos.mx)
}

\section{RESUMEN}

Actualmente existen opciones biológicamente sustentables que pueden sustituir parcialmente la fertilización sintética de los cultivos, disminuyendo los costos de producción. Para evaluar la respuesta de 18 variedades de maíz a la fertilización sintética (FQ), fertilización sintética más inoculante (FQB) y fertilización sintética más composta (FQC), en 2010 se establecieron dos experimentos en condiciones de temporal en el valle de Puebla, bajo un diseño completamente al azar con arreglo en parcelas divididas con tres repeticiones, a las parcelas grandes se les asignó el tipo de fertilización y en las parcelas chicas se integraron los genotipos de maíz. No se encontraron diferencias en rendimiento de grano y rastrojo por tipo de fertilización. Los resultados mostraron interacción de las variedades con los tipos de fertilización y localidad. En la interacción variedad por tipo de fertilización se encontró que el 55.6\% de las variedades mostró mayores rendimientos con $\mathrm{FQC}$, mientras que el $27.8 \%$ con FQB y el $16.7 \%$ con FQ. En San Andrés Calpan se obtuvieron los mayores rendimientos de rastrojo con FQC (8.8 $\left.\mathrm{Mg} \mathrm{ha}^{-1}\right)$ mientras que en San Pedro Tlaltenango estos se obtuvieron con la FQB (15.3 $\left.\mathrm{Mg} \mathrm{ha}^{-1}\right)$ y FQ (15.4 $\left.\mathrm{Mg} \mathrm{ha}^{-1}\right)$. El empleo combinado de fertilizante sintético con inoculantes o composta mantiene e incrementa los rendimientos de grano y rastrojo al obtenido sólo con la fertilización sintética.

Cita recomendada:

Alvarado Teyssier, R., E. Aceves Ruiz, J. D. Guerrero Rodríguez, J. I. Olvera Hernández, Á. Bustamante González, S. Vargas López y J. H. Hernández Salgado. 2018. Respuesta de variedades de maíz (Zea mays L.) a diferentes fuentes de fertilización en el Valle de Puebla. Terra Latinoamericana 36: 49-59. DOI: https://doi.org/10.28940/terra.v36i1.309
Palabras clave: genotipos, Azospirillum, inoculante, composta.

\section{SUMMARY}

Nowadays, there are biologically sustainable options that can partially replace synthetic fertilizers and reduce production costs. To test the response of 18 maize varieties to synthetic fertilization (FQ), synthetic fertilization plus inoculant (FQB) and synthetic fertilization plus compost (FQC), in 2010 two experiments were established in rainfed conditions in the Valley of Puebla, with a completely randomized design, under a split plots arrangement, with three replications. Type of fertilization was assigned to the main plot and maize genotypes were the split plot. No differences were found in grain and stover yield by type of fertilization. There was a strong interaction between varieties and type of fertilizer as well as between varieties and location in grain and stover yield. For the interaction between variety and type of fertilization, it was found that $56 \%$ of the varieties showed higher yields with FQC, in while $27.8 \%$ had higher yields with FQB and $16.7 \%$ yielded better with FQ. The highest stover yield was obtained in San Andrés Calpan with FQC $\left(8.8 \mathrm{Mg} \mathrm{ha}^{-1}\right)$, while in San Pedro Tlaltenango it was obtained with FQB (15.3 $\left.\mathrm{Mg} \mathrm{ha}^{-1}\right)$ and FQ (15.4 $\left.\mathrm{Mg} \mathrm{ha}^{-1}\right)$. The use of both synthetic fertilization and inoculant or compost maintains and increases maize grain and stover yield, compared with using only synthetic fertilizer.

Recibido: junio de 2017. Aceptado: diciembre de 2017. Publicado en Terra Latinoamericana 36: 49-59. 
Index words: genotypes, Azospirillum, bio-fertilizer, compost.

\section{INTRODUCCIÓN}

El maíz es el cultivo más importante de México, tanto por el volumen de producción como por la superficie cultivada. A su producción se dedica el $55 \%$ de la población total agropecuaria, encontrándose diversos estratos socioeconómicos que van desde los grandes empresarios hasta los agricultores de subsistencia (Polanco y Flores, 2008). En 2016 se cultivaron aproximadamente 7.6 millones de hectáreas obteniéndose un rendimiento promedio de $3.72 \mathrm{Mg} \mathrm{ha}^{-1}$ (SIAP, 2016). En este año a nivel nacional, Puebla se ubicó en el onceavo productor de maíz de México, con una producción de 1062000 toneladas (SIAP, 2016), cultivándose principalmente variedades nativas, según INEGI (2007), en el 2006, el 20\% de la superficie agrícola se cultivó con variedades mejoradas.

En el estado de Puebla, el Distrito de Desarrollo Rural (DDR) 05 Cholula es uno de los más importantes en la producción de este cultivo, aportando el 17.6 y $23 \%$ de la superficie cultivada y producción estatal, respectivamente (SIAP, 2016). En ese distrito los productores utilizan tanto el grano como el rastrojo, el primero para la alimentación humana y animal y el segundo exclusivamente para la alimentación del ganado. Es por ello que en el mejoramiento, que por años han realizado los productores a sus maíces nativos, han buscado que estos materiales produzcan cantidades adecuadas de grano y rastrojo.

En los agrosistemas con mayor potencial para la producción de maíz a partir del inicio del desmantelamiento de la Productora Nacional de Semillas (PRONASE) en 1994, se incrementó la oferta de variedades mejoradas de maíz, por la entrada de empresas extranjeras productoras de semillas, sin que mediara una evaluación de estas antes de ser recomendadas (Espinosa et al., 2010). Sin embargo, la mayoría de ellas no han sido aceptadas por los productores, ya que no satisfacen sus expectativas en la calidad y producción de grano y rastrojo (Márquez et al., 2000). De ahí que una gran cantidad de productores este en la búsqueda continua de variedades que presenten rendimientos adecuados, tanto de grano como de rastrojo.
Por otro lado, la producción del cultivo de maíz en el DDR 05 Cholula se sustenta en la utilización de agroquímicos, siendo el más importante los fertilizantes sintéticos. A partir de la década de los 90' el precio de estos ha sufrido incrementos constantes, a causa del desmantelamiento nacional de la industria productora de fertilizantes (FERTIMEX); y el incremento del precio de los hidrocarburos, materia prima para su elaboración (Ávila, 2001). Estos incrementos fueron mayores al $100 \%$ en el periodo de 2002-2008 (SNIIM, 2010), lo que ha afectado seriamente los costos de producción de este cultivo.

A pesar de la situación adversa en los costos de los insumos, en el estado de Puebla la superficie cultivada con maíz se ha mantenido; esto se debe principalmente a que la producción de este cultivo, en el estado y a nivel nacional, está soportada por los pequeños productores que siguen cultivando el maíz para asegurar su propia alimentación y no para la comercialización (Córdova, 2002). Para disminuir los costos de producción los productores han reducido la cantidad de fertilizante que aplican a sus cultivos, a sabiendas de que sus rendimientos se verán mermados. En el caso del Valle de Puebla, en la producción de maíz, un gran porcentaje de productores eliminó la aplicación de fertilizantes fosfatados y aplicó menor cantidad de fertilizantes nitrogenados ${ }^{1}$. Ante esta situación, el productor demanda nueva tecnología que no implique fuertes inversiones y que incida significativamente en los rendimientos de maíz, no sólo en el incremento de la producción de grano, sino también en el rastrojo, ya que este último forma parte fundamental en la alimentación de su ganado, el cual representa una fuente importante en su ingreso familiar, además de mejorar la rentabilidad del cultivo.

La producción de maíz puede lograrse de manera sustentable con el uso de inoculantes y fertilizantes orgánicos, además de reducir significativamente los costos de producción y mantener los niveles de producción sin deteriorar el medio ambiente (Bashan et al., 1997; Morales, 2007). Al respecto se han reportado efectos significativos en el rendimiento de grano de diversos cultivos con la inoculación con Azospirillum. García et al. (2012) señalan que la inoculación con Azospirillum incrementa hasta en $35 \%$ el rendimiento de maíz respecto a plantas no inoculadas, Mientras que Dobbelaere et al. (2001),

${ }^{1}$ Comunicación Personal Rafael Alvarado Teyssier. Información obtenida a través de una encuesta en el área de estudio sin publicar. 
Diaz et al. (2009) y Hungria et al. (2010) señalan que la inoculación con esta bacteria puede incrementar los rendimientos de maíz, trigo, sorgo, arroz y leguminosas ente un 5 y 30\%. De acuerdo a Helman et al. (2011) la dosis de fertilizante nitrogenado puede reducirse entre 20 y 50\% con la inoculación de Azospirillum. Por otra parte, Matheus (2004) señala que la aplicación de una mezcla de fertilizante químico y orgánico es una alternativa para la producción de maíz; así mismo, Álvarez et al. (2010) y Uribe et al. (2007), sugieren un manejo integral de la fertilización, es decir, no solo aplicar mezclas de fertilizantes químicos y orgánicos, sino complementarlos con inoculantes para tener una mejor respuesta del cultivo.

Por lo antes señalado, en el presente trabajo evaluó la respuesta de variedades de maíz a tres tipos de fertilización.

\section{MATERIALES Y MÉTODOS}

En el ciclo agrícola 2010 en condiciones de temporal se establecieron dos experimentos de campo localizados en $19^{\circ} 10^{\prime} 15.45^{\prime \prime} \mathrm{N}$ y $98^{\circ} 21^{\prime} 13.82$ " O, con altitud de $2228 \mathrm{~m}$ en la localidad de San Pedro Tlaltenango y otro a $19^{\circ} 06^{\prime} 54.43^{\prime \prime} \mathrm{N}$ y $98^{\circ} 22^{\prime} 57.61^{\prime \prime} \mathrm{O}$, con una altitud de 2246 m en San Andrés Calpan en el estado de Puebla. Se determinaron algunas características físico-químico de los suelos de cada sitio (Cuadro 1). Se evaluó la respuesta de 18 variedades de maíz a la fertilización sintética (FQ), FQ más inoculante (FQB) y FQ más composta (FQC).

El tratamiento de FQ consistió en la aplicación de 160-70-00 para $\mathrm{N}, \mathrm{P}_{2} \mathrm{O}_{5}$ y $\mathrm{K}_{2} \mathrm{O}$ respectivamente. $\mathrm{La}$ FQB consistió en la aplicación de la fórmula 80-35-00 para $\mathrm{N}, \mathrm{P}_{2} \mathrm{O}_{5}$ y $\mathrm{K}_{2} \mathrm{O}$ respectivamente y los inoculantes biofertibuap y biofosfobuap, el primero formado a base de la bacteria Azospirillum brasilenses y biofosfobuap contiene a las bacterias Acinetobacter calcoaceticus y Chromobacterium violaceum.
La FQC estuvo integrada de la fórmula 80-35-00 para $\mathrm{N}, \mathrm{P}_{2} \mathrm{O}_{5}$ y $\mathrm{K}_{2} \mathrm{O}$ respectivamente y de $3.5 \mathrm{Mg} \mathrm{ha}^{-1}$ de lombricomposta de cachaza de caña con las siguientes características físico-químicas: $\mathrm{pH}$ 8.29, CE $11.34 \mathrm{dsm}^{-1}, 12.77,0.81,0.35,0.63,2.58 \mathrm{y}$ $0.24 \%$ de $\mathrm{MO}, \mathrm{N}, \mathrm{P}, \mathrm{K}, \mathrm{Ca}$ y $\mathrm{Mg}$ respectivamente y $27.1 \mathrm{cmol} \mathrm{kg}^{-1}$ de CIC. En todos los tratamientos un tercio del $\mathrm{N}$, todo el $\mathrm{P}, \mathrm{K}$, inoculante y composta se aplicaron en la siembra, el resto de nitrógeno se aplicó a los 50 días después de la siembra. En la fertilización sintética se utilizó urea como fuente de nitrógeno y fosfato diamónico para fósforo.

Se evaluaron 18 variedades de maíz recomendados para la región de estudio (Cuadro 2).

Se utilizó un diseño experimental de bloques al azar con un arreglo en parcelas divididas con tres repeticiones. La parcela grande (PG) correspondió a tratamientos de fertilización (FQ, FQC, FQB), mientras en la parcela chica (PD) se ubicó al factor variedad. Cada parcela grande constó de 36 surcos de cinco metros de largo y $0.90 \mathrm{~m}$ de ancho. A cada parcela chica (variedad) se le asignaron dos surcos. La densidad de población fue de 60000 plantas ha-1 con un arreglo topológico de dos plantas por mata. Al momento de la siembra se depositaron tres semillas por mata, a los 15 días se realizó el aclareo de plantas dejando dos plantas por mata.

La cosecha se realizó una vez que el cultivo alcanzó la madurez fisiológica, cosechando sólo aquellas plantas que presentaban competencia completa. De cada parcela chica, se tomó una muestra de cinco mazorcas y tres plantas para determinarles el contenido de humedad. El rendimiento de grano y rastrojo se calculó con $14 \%$ de humedad. Los resultados de rendimientos de grano y rastrojo por parcela chica se analizaron con una prueba de varianza combinado de acuerdo al modelo estadístico para experimentos en serie (Martínez, 1988), con el programa Statistical Analysis System (SAS) versión 9.1 (SAS Institute,

Cuadro 1. Características físico-químicas de los suelos de cada sitio experimentales.

\begin{tabular}{|c|c|c|c|c|c|c|c|c|c|c|c|}
\hline Localidad & $\mathrm{pH}$ & MO & $\mathrm{P}$ & $\mathrm{K}$ & $\mathrm{Ca}$ & $\mathrm{Mg}$ & $\begin{array}{c}\text { Acidez } \\
\text { intercambiable }\end{array}$ & Arena & Limo & Arcilla & Textura \\
\hline & & $\%$ & $-\cdots$ & $-\cdots n$ & $\mathrm{~kg}^{-1}-$ & $-\ldots$ & $\mathrm{cmol} \mathrm{kg}^{-1}$ & $-\ldots$ & $-\%$ & $-\cdots$ & \\
\hline San Pedro Tlaltenango & 6.47 & 0.6 & 23.21 & 228 & 843 & 405 & 0.61 & 74.2 & 16 & 9.8 & Franco arenoso \\
\hline San Andrés Calpan & 6.03 & 0.47 & 24.23 & 126 & 243 & 183 & 1.01 & 61.5 & 32.7 & 5.8 & Franco arenoso \\
\hline
\end{tabular}


Cuadro 2. Origen y variedades de maíz utilizadas en el estudio.

\begin{tabular}{lll}
\hline Variedad $^{\dagger}$ & Estatus & Origen \\
\hline $1,2,3,4,5,6,7,8,9,10$ & Evaluación & CP-Campus Montecillo \\
164,167 & Evaluación & CP-Campus Puebla \\
HS2 & Mercado & CP-Campus Montecillo \\
Sintético Serdán (SSER) & Mercado & CP-Campus Puebla \\
AS822 & Mercado & ASPROS \\
32 D06 & Mercado & PIONEER \\
Niebla & Mercado & CERES \\
Nativo & & Productor cooperante
\end{tabular}

${ }^{\dagger}$ Las variedades del 1 al 10 se identificaron de esta manera para facilitar su manejo, debido a que aún no tienen nombre. $\mathrm{CP}=$ Colegio de Postgraduados.

2003). A los factores de estudio que presentaron significancia estadística se les realizó la prueba de medias de Tukey.

\section{RESULTADOS Y DISCUSIÓN}

Los resultados de rendimiento de grano y rastrojo fueron altamente significativos en los factores localidad, variedad, y las interacciones localidad $\times$ variedad, fertilización $\times$ variedad y localidad $\times$ fertilización $\times$ variedad $(P<0.0001)$; para el resto de los factores (fertilización y localidad $\times$ fertilización) el rendimiento de grano no fue significativamente diferente; en tanto que para el rendimiento de rastrojo la respuesta fue significativa para la interacción localidad $\times$ fertilización $(P<0.0001)$; mientras que para el factor fertilización el efecto no fue significativo.

\section{Respuesta al Factor Localidad}

El rendimiento de grano y rastrojo de maíz fueron mayores en la localidad de San Pedro Tlaltenango (Cuadro 3), lo cual supera en 38.4 y $46.4 \%$ el rendimiento de grano y rastrojo respectivamente al obtenido en San Andrés Calpan. Estas diferencias obedecen posiblemente a que en San pedro Tlaltenango hubo mayor precipitación (119.3 mm más que en San Andrés Calpan) lo que permitió que se presentará un mayor porcentaje de plantas cuateras, $50 \%$ más que en San Andrés Calpan; además pudo haber influido que el suelo presentó mayores contenidos de $\mathrm{K}, \mathrm{Ca}$ y $\mathrm{Mg}$ que los del suelo de Tlatenango. Resultados semejantes reporta Lerma (1995) entre la localidad de San Mateo
Cuadro 3. Rendimiento promedio de grano y rastrojo por localidad.

\begin{tabular}{lcc}
\hline \multirow{2}{*}{ Localidad } & \multicolumn{2}{c}{ Rendimiento } \\
\cline { 2 - 3 } & Grano & Rastrojo \\
\hline \multirow{2}{*}{ San Pedro Tlaltenango } & $10.486 \mathrm{a}$ & $15.029 \mathrm{a}$ \\
San Andrés Calpan & $6.458 \mathrm{~b}$ & $8.056 \mathrm{~b}$ \\
$\mathrm{DSH}$ & 0.341 & 3.238 \\
\hline Medias con la misma letra sobre la columna no presentan diferencias \\
significativas. DSH = diferencia significativa honesta.
\end{tabular}

Capultitlán y San Andrés Calpan, la primera con condiciones semejantes a las de San Pedro Tlaltenango.

\section{Respuesta a Fertilización}

En relación a la respuesta a tipo de fertilización no se encontraron diferencias estadísticamente significativas para rendimiento de grano y rastrojo, lo que indica que en la zona de estudio se obtiene el mismo rendimiento de grano y rastrojo con cualquiera de los tratamientos de fertilizante evaluados, lo que demuestra que es posible reducir la dosis de fertilización química con la utilización de otros insumos (lombricomposta o Azospirillum con Acinetobacter calcoaceticus y Chromobacterium violaceum ) sin que merme el rendimiento, lo que se buscaba en el presente estudio. Al respecto, Kimetu et al. (2004) y Matheus (2004), obtuvieron mayores rendimientos de grano de maíz con la fertilización combinada de productos orgánicos y fertilización química que los obtenidos cuando estos se aplicaron por separado; por otro lado, Irizar et al. (2003) encontraron rendimientos similares en el cultivo de maíz con la aplicación de fertilizante sintético más Azospirillum y fertilización sintética con Azospirillum más micorrizas al obtenido al aplicar solo fertilización sintética.

\section{Respuesta a la Interacción Localidad por Fertilización}

Se encontró diferencia estadísticamente significativa de la interacción localidad $\times$ fertilización para el rendimiento de rastrojo. La diferencia en rendimiento de rastrojo entre ambas localidades con la FQ fue de $8 \mathrm{Mg} \mathrm{ha}^{-1}$; mientras que con $\mathrm{FQB}$ fue de 7.4 Mg ha ${ }^{-1} \mathrm{y}$ con FQC fue de 5.5 $\mathrm{Mg} \mathrm{ha}^{-1}$ (Figura 1), 


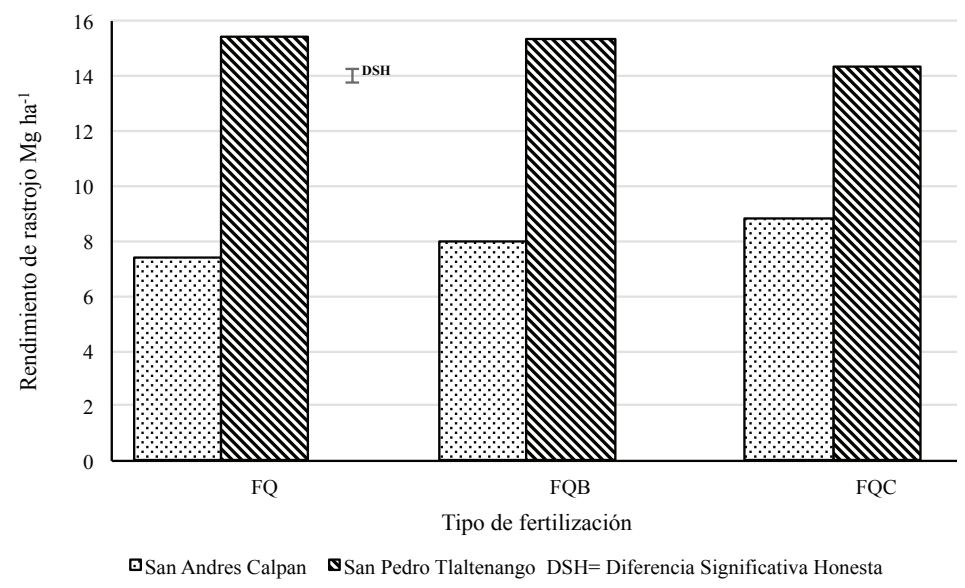

Figura 1. Rendimiento de rastrojo de maíz por localidad y tipo de fertilización. $\mathrm{FQ}=$ Fertilización quimica, $\mathrm{FQB}=\mathrm{FQ}$ más inoculante, $\mathrm{FQC}=\mathrm{FQ}$ más composta.

en todos los casos estas diferencias son a favor de la localidad de San Pedro Tlaltenango, donde el mayor rendimiento promedio se obtuvo con el manejo de fertilización sintética y el menor rendimiento con FQC. En Calpan el mayor rendimiento se obtuvo utilizando FQC y el menor con FQ. La diferencia se podría atribuir a la disimilitud de la precipitación entre las localidades, en Tlaltenango llovió $119.3 \mathrm{~mm}$ más que en Calpan lo que pudo haber favorecido que se diera un mayor aprovechamiento de la FQ, en tanto que en Calpan, la composta pudo haber favorecido la mejora de las propiedades fisico-químicas del suelo que permitieron un mejor aprovechamiento de la humedad, tal como lo señalan Whalen et al. (2003) y Arancon y Edwards (2005) quienes mencionan que la utilización de compostas mejoran las propiedades físico-químicas y biológicas del suelo lo cual favorece el desarrollo de los cultivos.

\section{Respuesta a Variedad}

La diferencia entre variedades de maíz en rendimiento de grano y rastrojo fue altamente significativa. Las variedades con mayor rendimiento de grano fueron la 9, 10.3 Mg ha' ${ }^{-1}$ HS-2, $10.1 \mathrm{Mg} \mathrm{ha}^{-1}$; AS822, 10.1 $\mathrm{Mg} \mathrm{ha}^{-1}$; 32D06, $9.8 \mathrm{Mg} \mathrm{ha}^{-1}$; y niebla, 9.5 $\mathrm{Mg} \mathrm{ha}^{-1}$; mientras que las que mostraron menor rendimiento fueron 164, 4.4 $\mathrm{Mg} \mathrm{ha}^{-1}$; $167,6.2 \mathrm{Mg} \mathrm{ha}^{-1}$; Sintético Serdán, $6.7 \mathrm{Mg} \mathrm{ha}^{-1}$;, $7.6 \mathrm{Mg} \mathrm{ha}^{-1} \mathrm{y}$ nativo, $8.3 \mathrm{Mg} \mathrm{ha}^{-1}$ (Figura 2). La diferencia entre

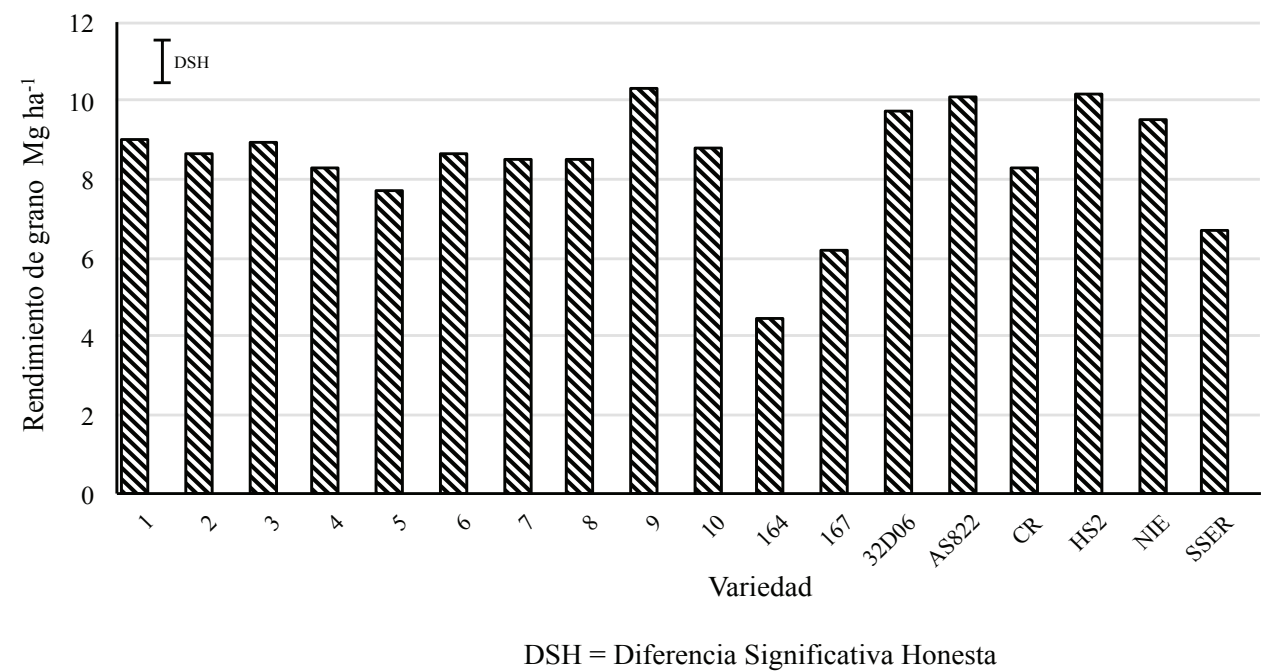

Figura 2. Rendimiento de grano de maíz por variedad. 
las variedades evaluadas puede ser atribuible a que las variedades con mayor rendimiento tienen un fuerte trabajo de mejoramiento genético, mientras que las tres variedades que mostraron los menores rendimientos, son selecciones sobresalientes de materiales nativos, estos últimos presentaron un alto porcentaje de acame en la etapa de floración (29.8\% contra $10.6 \%$ de las variedades con mayor rendimiento), lo que provocó la presencia de plantas jorras o sin mazorca (23\% contra $4.35 \%$ de las variedades más rendidoras). El acame en la etapa de floración y llenado de grano reduce el rendimiento de grano (Wiersma et al., 1986; González y Larios, 1993; Niño et al., 1998), por restringir entre otras cosas la fecundación y la traslocación de fotosintatos al grano.

El rendimiento de rastrojo fue más alto en las variedades 167 , nativo, 164,8 , HS-2 y Sintético Serdán; mientras que las variedades 32D06, 10, 6 y 4 fueron las que presentaron los menores rendimientos (Figura 3). El hecho de que las variedades 167, 164 y sintético Serdán se encuentren entre las variedades con mayor rendimiento de rastrojo puede deberse a que al presentarse un gran porcentaje de plantas sin mazorca los fotosintatos que estas producían las destinaron a la producción de paja (Jugenheimer, 1990).

En la zona de estudio, la producción de rastrojo juega un papel importante en el ingreso de la unidad de producción familiar, ya que el productor lo utiliza como alimento para sus animales o bien lo comercializa en pacas de aproximadamente $35 \mathrm{~kg}$, entero o molido, a productores con establos. Por esta razón se buscan variedades que produzcan cantidades adecuadas y estables a través de los años de grano y rastrojo, por lo que no es suficiente que la variedad produzca cantidades suficientes de estos dos productos, el grano debe de cumplir ciertas características para la producción de tortilla, mientras que el rastrojo debe cumplir con características de palatabilidad para los animales.

\section{Respuesta a la Interacción Localidad por Variedad}

Al comparar entre localidades todas las variedades presentaron mayores rendimientos en la localidad de Tlaltenango (Figura 4). El comportamiento de las variedades dependió de la localidad, en Calpan las variedades que prestaron mayor rendimiento fueron niebla, 9 y 32D06, mientras que en Tlaltenango fueron AS822, HS-2 y 9. Se observó que las variedades que presentaron mayor interacción con el ambiente fueron la 8, A822, SSER, HS2, y 164 ya que disminuyeron sus rendimientos en $44.8,45.9,47.7,50.1$ y $50.5 \%$ respectivamente en la comunidad de calpan con respecto al rendimiento obtenido en Tlaltenengo. En el área de estudio se han realizado diversos trabajos que han mostrado una fuerte interacción entre localidades y variedades, Gil et al. (2004), evaluaron diferentes genotipos nativos y encontraron diferencia en la producción de grano altamente significativa entre localidades y para la interacción localidades

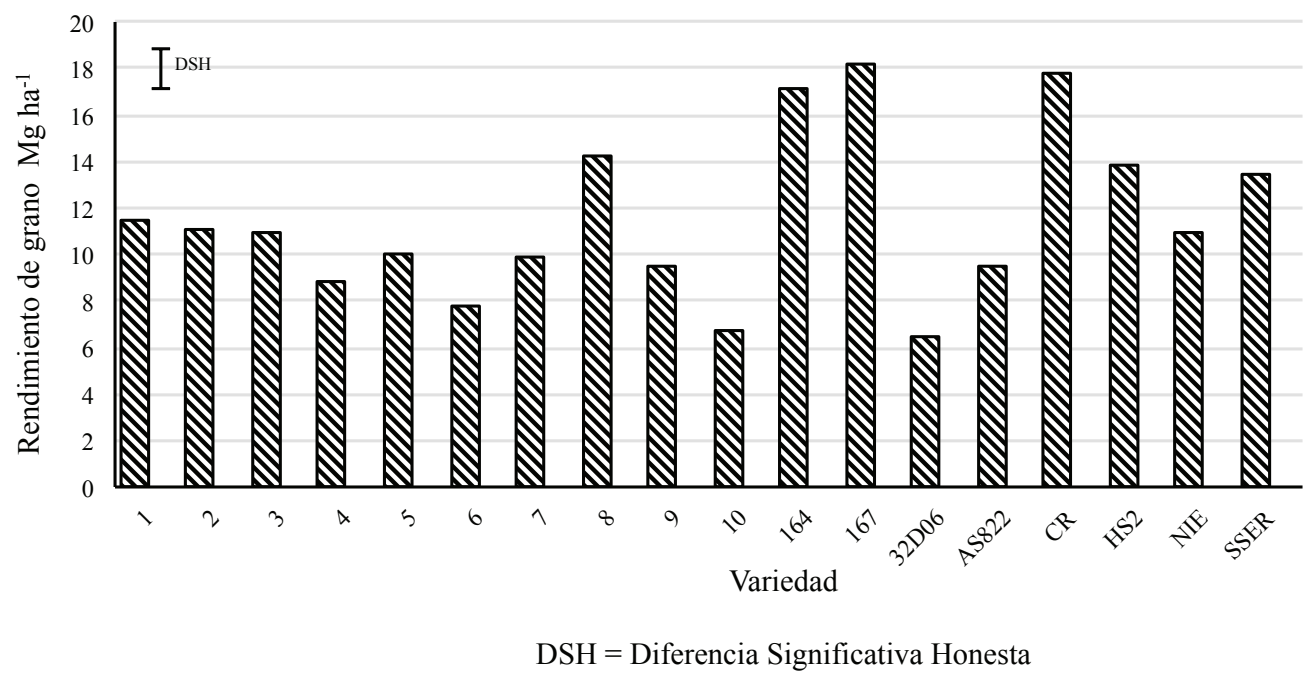

Figura 3. Rendimiento de rastrojo de maíz por variedad. 


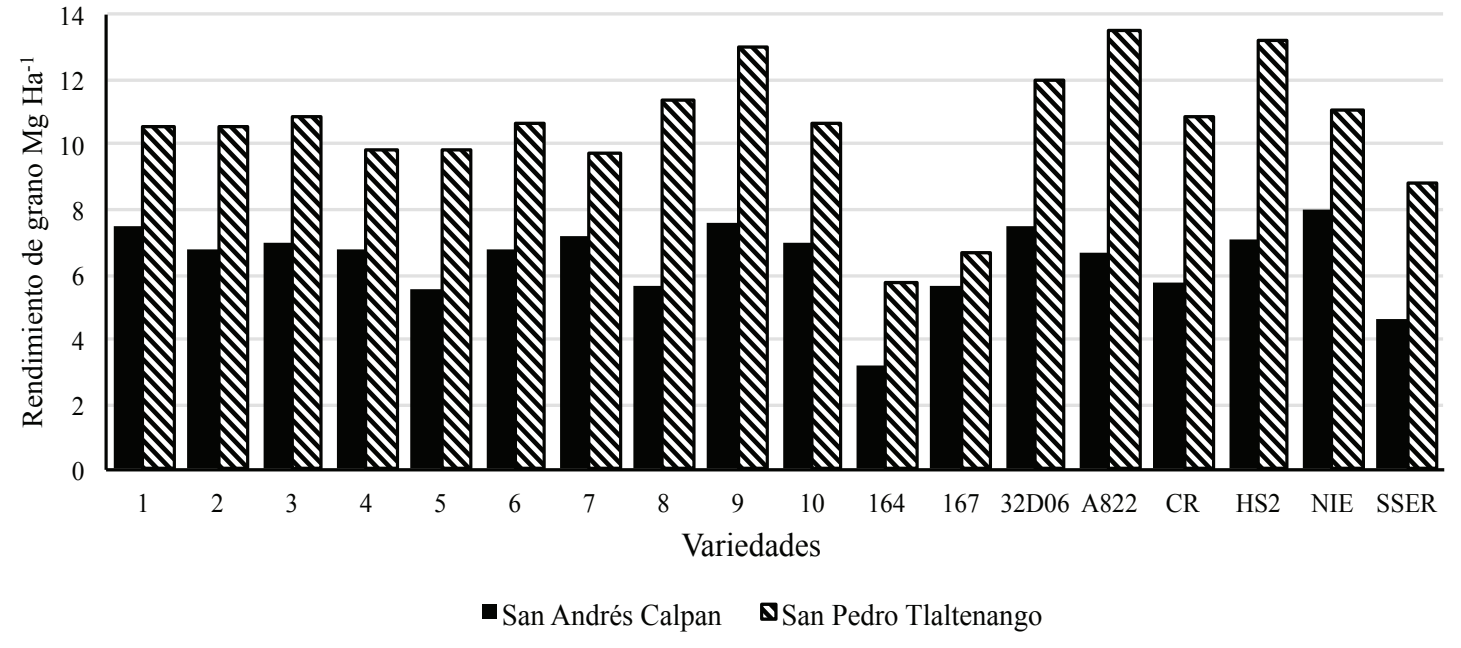

Figura 4. Rendimiento de grano de maíz para interacción localidad por variedad.

$\times$ variedades. Aceves et al. (2002) encontraron interacciones significativas entre localidad y genotipo, entre variedades nativas y el híbrido H-137.

En cuanto a rendimiento de rastrojo se observó que todas las variedades evaluadas, excepto la 10, presentaron mayores rendimientos en la localidad de Tlaltenango (Figura 5).

Las variedades que presentaron mayor efecto en el rendimiento de rastrojo al sembrarse en la localidad de calpan fueron la 5, 2, 6, A822, 8, HS2 y 164 disminuyendo su rendimiento en 63.7, 61.1, 59.6, 57.4, $57.3,56.1$ y $55.0 \%$ respectivamente, con respecto al rendimiento obtenido en Tlaltenango.

\section{Respuesta a la Interacción Tipo de Fertilización por Variedad}

El $55.6 \%$ de las variedades mostró mayores rendimientos de grano con el tratamiento $\mathrm{FQC}$, mientras que el $27.8 \%$ lo hizo con FQB, y el $16.7 \%$ con FQ. El $38.9 \%$ de las variedades presentaron los menores rendimientos de grano al ser manejadas con FQ y FQB, en tanto que el $22.2 \%$ de las variedades presentaron los menores rendimientos con la FQC (Figura 6). De las cuatro variedades que mayor cantidad de grano produjeron, tres de ellas (32D06, HS-2 y

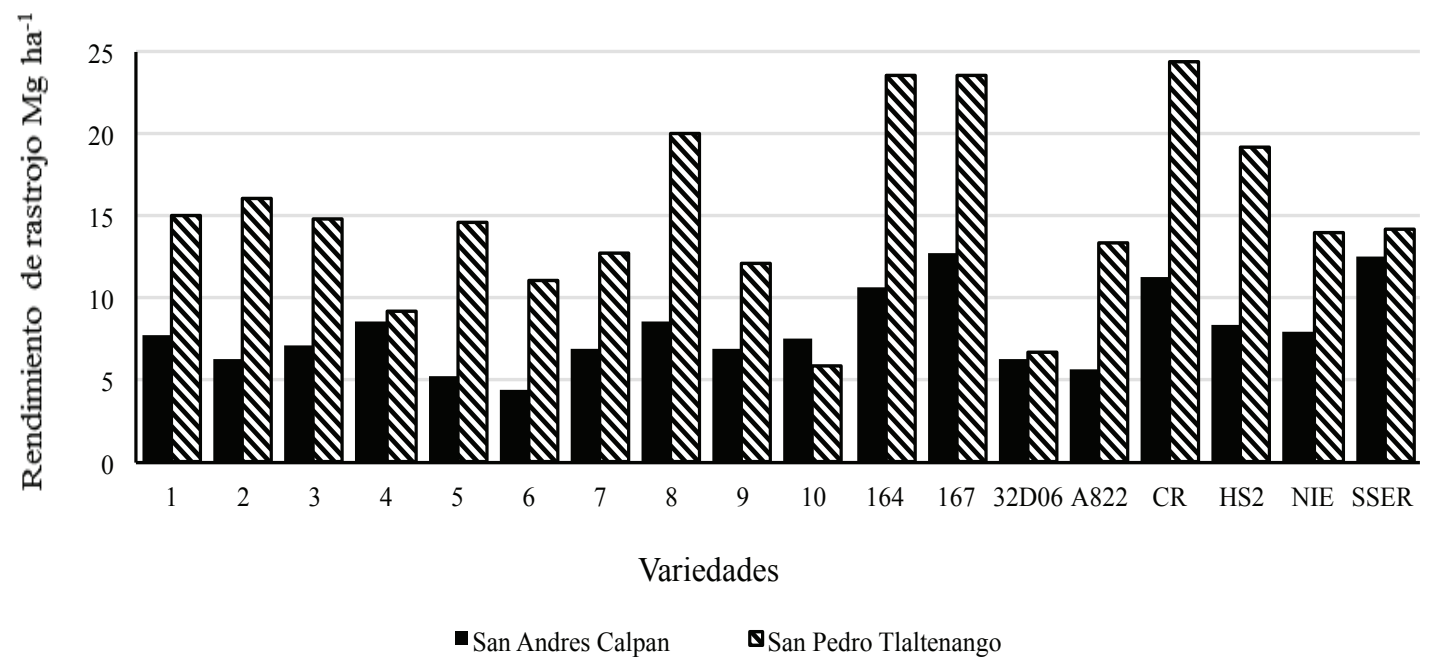

Figura 5. Rendimiento de rastrojo de maíz por la interaccion localidad por variedad. 
AS822) lo hicieron con la FQC y una (9) con la FQB. Estos resultados nos indican que el tipo y cantidad de fertilizante a aplicar va a depender de la variedad que el productor emplee.

En el rendimiento de rastrojo el número de variedades que presentaron los rendimientos más altos por tipo de fertilizante estuvo equilibrado con $33.33 \%$ de las variedades por tipo de fertilización, en tanto que el menor rendimiento de rastrojo se obtuvo con la FQ (Figura 7). Las variedades 4 y 3 fueron las únicas que presentaron el rendimiento más alto, tanto de grano como de rastrojo, con la fertilización sintética; mientras que con la $\mathrm{FQB}$ solo fue la variedad nativa; y con la FQC coinciden las variedades 10, 32D06 y Niebla. Los resultados muestran que dependiendo de la variedad a producir será el tipo de fertilización que se recomendará. Por otro lado, entre las cuatro variedades que produjeron la mayor cantidad de rastrojo, la $167 \mathrm{y}$ nativo lo hicieron con la FQB, la 164 con la FQC y el Sintético Serdán con la fertilización sintética.

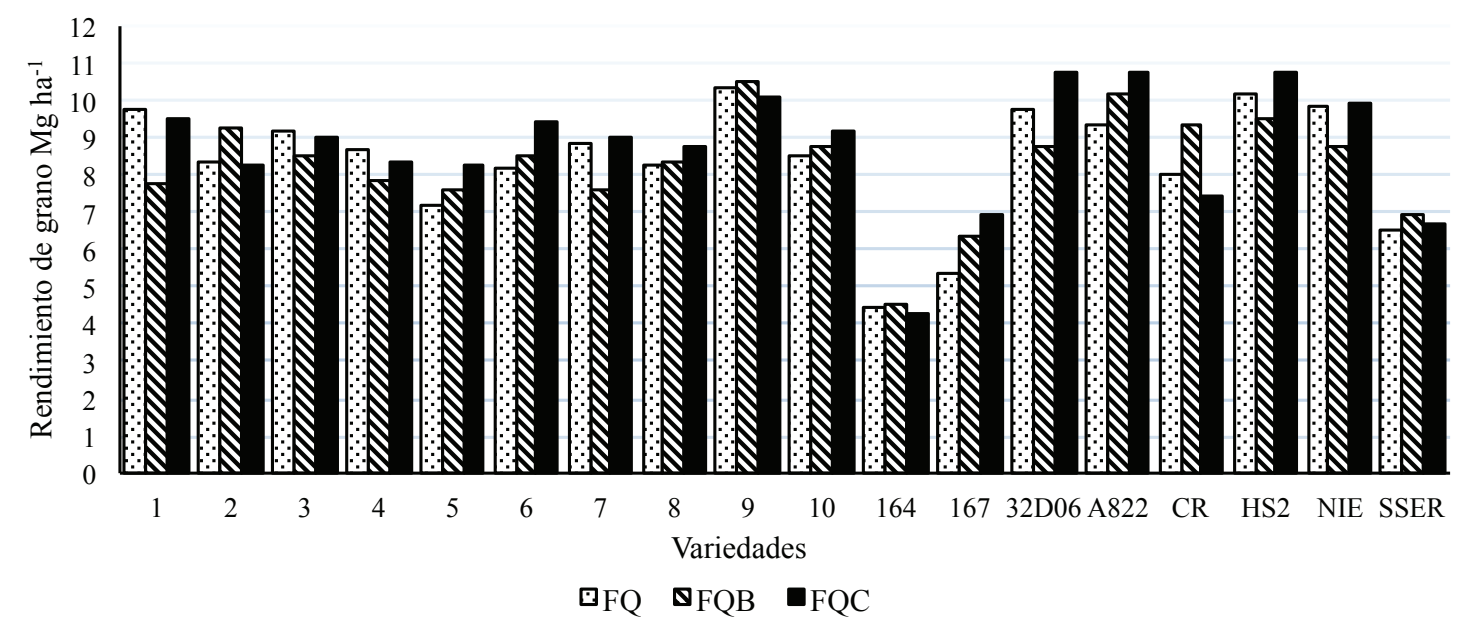

Figura 6. Rendimiento de grano de maíz por la interacción tipo de fertilizante por variedad.

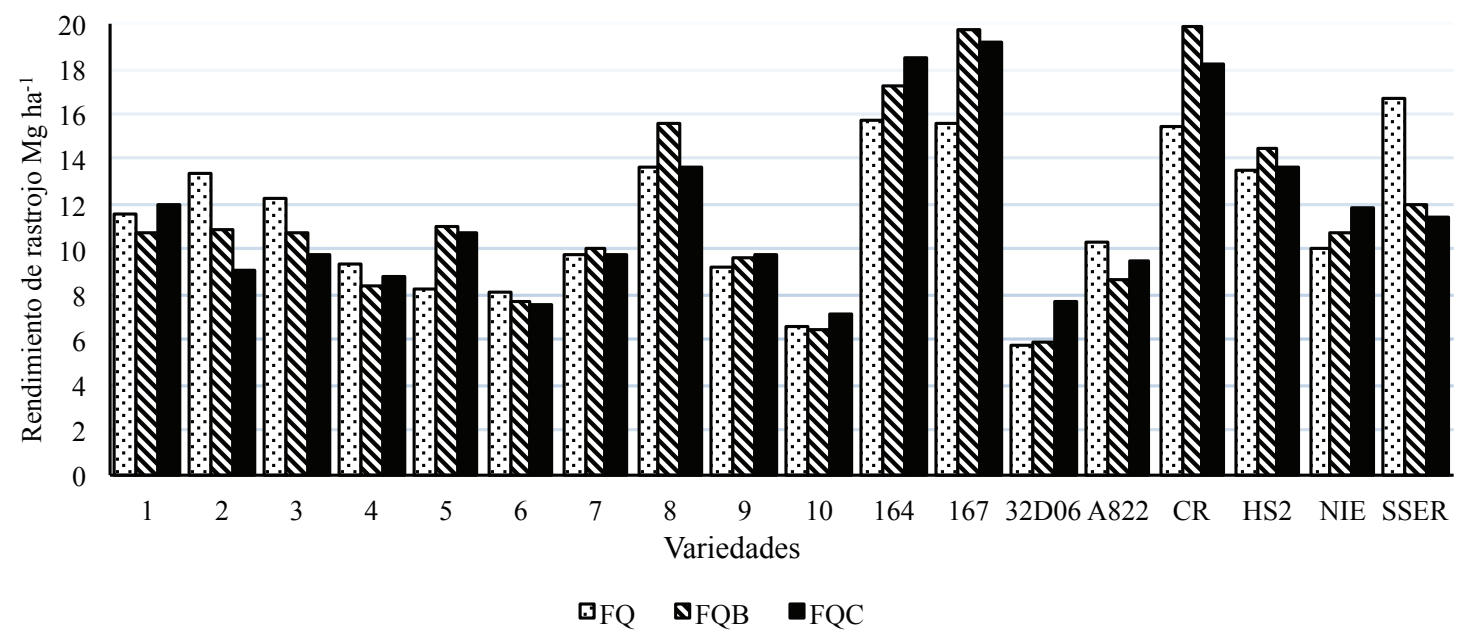

Figura 7. Rendimiento de rastrojo de maíz por la interacción tipo de fertilizante por variedad. 


\section{Respuesta a la Interacción Localidad por Tipo de Fertilización por Variedad}

El rendimiento de grano y rastrojo en la interacción localidad $\times$ tipo de fertilización $\times$ variedad presentó diferencias altamente significativas. Se encontró que en Calpan las dos variedades que presentaron mayor rendimiento fueron la 32D06 y niebla con el tratamiento FQC, mientras que en Tlaltenango la variedad AS822 con FQB y HS2 con la FQ (Cuadro 4).

En cuanto a rastrojo, de manera general, se observó que las variedades que presentaron los menores rendimientos de grano son los que muestran los mayores rendimientos de rastrojo en ambas localidades y los tres tipos de fertilización (Cuadro 4). En Calpan los mayores rendimientos de rastrojo se obtuvieron con las variedades sintético sedán con $\mathrm{FQC} \mathrm{y} \mathrm{nativo}$ con $\mathrm{FQB}$, en tanto que, en Tlaltenango los mayores rendimientos de rastrojo se obtuvieron con la FQC y las variedades 167 y 164 . Los resultados reflejan las diferencias genéticas de las variedades para la respuesta a los factores evaluados, ya que de acuerdo a Lafitte y Edmeades (1991) y Zobel (1991) citado por Barrios et al. (2008) el rendimiento potencial de los genotipos se debe al manejo, resistencia de factores bióticos y tensiones ambientales.

La fuerte interacción que se presenta entre estos factores nos indica que, al momento de introducir una variedad a una región, se debe delimitar el área y el tipo de fertilizante con el que tiene mejor comportamiento, cosa que en la actualidad no se realiza, quizás por la falta de recursos económicos para las investigaciones pertinentes.

Cuadro 4. Rendimiento de grano y rastrojo de maíz por localidad tipo de fertilización y variedad.

\begin{tabular}{|c|c|c|c|c|c|c|c|c|c|c|c|c|}
\hline \multirow{4}{*}{ Variedades } & \multicolumn{6}{|c|}{ Tlaltenango } & \multicolumn{6}{|c|}{ Calpan } \\
\hline & \multicolumn{3}{|c|}{ Rendimiento de grano } & \multicolumn{3}{|c|}{ Rendimiento de rastrojo } & \multicolumn{3}{|c|}{ Rendimiento de grano } & \multicolumn{3}{|c|}{ Rendimiento de rastrojo } \\
\hline & $\ldots$ & $\ldots$ & $-\cdots M$ & $a^{-1}-\cdots$ & $-\ldots$ & $-\ldots$ & $\ldots-$ & $\ldots$ & $--\mathrm{N}$ & $a^{-1}-\cdots$ & $\ldots$ & $-\ldots$ \\
\hline & $\mathrm{FQ}^{\dagger}$ & FQB & FQC & FQ & FQB & FQC & FQ & FQB & FQC & FQ & FQB & FQC \\
\hline 1 & 10.603 & 9.184 & 11.878 & 14.340 & 15.543 & 15.309 & 8.944 & 6.342 & 7.233 & 8.794 & 5.930 & 8.576 \\
\hline 2 & 10.272 & 11.645 & 9.819 & 20.634 & 16.093 & 11.387 & 6.464 & 6.929 & 6.799 & 6.139 & 5.672 & 6.898 \\
\hline 3 & 11.116 & 10.306 & 11.185 & 16.920 & 15.141 & 12.263 & 7.215 & 6.767 & 6.838 & 7.632 & 6.374 & 7.313 \\
\hline 4 & 9.972 & 9.227 & 10.241 & 9.488 & 8.493 & 9.476 & 7.423 & 6.528 & 6.392 & 9.166 & 8.336 & 8.221 \\
\hline 5 & 9.976 & 9.297 & 10.129 & 12.520 & 16.524 & 15.022 & 4.418 & 5.997 & 6.353 & 4.110 & 5.483 & 6.380 \\
\hline 6 & 9.590 & 10.177 & 12.141 & 11.799 & 10.902 & 10.578 & 6.739 & 6.804 & 6.653 & 4.369 & 4.575 & 4.510 \\
\hline 7 & 10.430 & 8.707 & 10.224 & 12.394 & 13.344 & 12.379 & 7.332 & 6.430 & 7.743 & 7.110 & 6.817 & 7.140 \\
\hline 8 & 11.470 & 11.199 & 11.345 & 19.676 & 22.493 & 17.901 & 5.013 & 5.569 & 6.245 & 7.590 & 8.646 & 9.405 \\
\hline 9 & 12.693 & 13.367 & 12.985 & 11.301 & 12.650 & 12.434 & 8.063 & 7.609 & 7.266 & 7.089 & 6.608 & 6.988 \\
\hline 10 & 10.567 & 10.693 & 10.555 & 6.030 & 6.068 & 5.665 & 6.434 & 6.883 & 7.754 & 7.015 & 6.765 & 8.571 \\
\hline 164 & 6.287 & 5.363 & 5.517 & 21.672 & 23.731 & 25.557 & 2.657 & 3.787 & 3.041 & 9.858 & 10.729 & 11.326 \\
\hline 167 & 5.996 & 6.461 & 7.707 & 19.835 & 24.255 & 26.606 & 4.676 & 6.248 & 6.109 & 11.373 & 15.275 & 11.654 \\
\hline $32 \mathrm{D} 06$ & 13.686 & 11.360 & 10.957 & 7.381 & 6.981 & 5.665 & 5.781 & 6.147 & 10.598 & 4.041 & 4.841 & 9.808 \\
\hline AS822 & 12.384 & 14.611 & 13.410 & 15.033 & 11.947 & 13.010 & 6.298 & 5.796 & 8.065 & 5.675 & 5.278 & 6.071 \\
\hline $\mathrm{CR}$ & 11.016 & 11.467 & 10.048 & 23.512 & 24.148 & 25.377 & 5.058 & 7.255 & 4.862 & 7.278 & 15.505 & 11.050 \\
\hline HS2 & 14.460 & 11.938 & 13.071 & 20.101 & 20.791 & 16.873 & 5.871 & 7.079 & 8.417 & 6.991 & 8.070 & 10.307 \\
\hline NIE & 11.662 & 10.937 & 10.445 & 12.943 & 14.134 & 14.663 & 7.988 & 6.595 & 9.416 & 7.144 & 7.459 & 9.168 \\
\hline SSER & 8.840 & 9.134 & 8.468 & 21.933 & 13.264 & 7.404 & 4.134 & 4.780 & 4.918 & 11.581 & 10.814 & 15.527 \\
\hline
\end{tabular}

${ }^{\dagger} \mathrm{FQ}$ = fertilización química; $\mathrm{FQB}=$ fertilización química más inoculante, $\mathrm{FQC}$ = fertilización química más composta. 


\section{CONCLUSIONES}

-Las variedades de maíz responden de diferente manera al tipo de fertilizante en cada uno de los ambientes evaluados, por lo que cuando se introduzca una nueva variedad en alguna región sería deseable determinar el tipo de fertilización que se le debe de aplicar.

- En la Localidad de San Pedro Tlaltenango el mejor tipo de fertilización para la producción de grano con las variedades utilizadas fue la fertilización sintética más inoculante y para la producción de rastrojo fue la fertilización sintética más composta. Mientras que en la localidad de San Andrés Calpan la mayor producción de grano y rastrojo se obtuvo con la fertilización sintética más composta.

-Las variedades que mostraron mayores rendimientos de grano presentaron los menores rendimientos de rastrojo.

- Al utilizar fertilizante sintético más inoculantes o composta es posible mantener e inclusive incrementar los rendimientos de grano y rastrojo de maíz en comparación con el manejo solo con la fertilización sintética, por lo que es factible reducir la cantidad de fertilizante sintético y complementarlo con inoculante o lombricomposta.

- En las dos localidades los genotipos de maíz que superan a los nativos en la producción de grano son las variedades 9, HS-2, AS822, 32D06 y Niebla; mientras que en la producción de rastrojo ningún material supera al maíz nativo.

\section{LITERATURA CITADA}

Aceves R., E., A. Turrent F., J. I. Cortés F. y V. Volke H. 2002. Comportamiento agronómico del híbrido $\mathrm{H}-137$ y materiales criollos de maíz en el Valle de Puebla. Rev. Fitotec. Méx. 25: 339-347.

Álvarez S., J. D., D. A. Gómez V., N. S. León M. y F. A. Gutiérrez M. 2010. Manejo integrado de fertilizantes y abonos orgánicos en el cultivo de maíz. Agrociencia. 44: 575-586.

Arancon, N. Q. and C. A. Edwards. 2005. Effects of vermicomposts on plant growth. pp. 1-25. In: Paper presented during the International Symposium Workshop on Vermi Technologies for Developing Countries (ISWVT 2005). Los Banos, Philippines November 16-18. ISBN: 978-971926-091-2.

Ávila, J. A. 2001. El mercado de los fertilizantes en México/ situación actual y perspectivas. Prob. Desarrollo: 32: 189-207.

Barrios A., A., A. Turrent F., R. Ariza F., M. Otero S. y A. Michel A. 2008. Interacción genotipo $\mathrm{X}$ prácticas de manejo en el rendmiento de grano de híbridos de maíz. Agric. Téc. Méx. 34: 85-90.
Bashan Y. and G. Holguín. 1997. Azospirillum - plant relationships: Environmental and physiological advances (1990-1996). Can. J. Microbiol. 43: 103-121.

Córdova, H. 2002. El efecto de la globalización sobre los programas de fitomejoramiento de las instituciones públicas y perspectivas para la autosuficiencia en maíz hacia el año 2020. CIMMYT, INT. México. pp.1 -17. In: J. M. Martínez R., F. Rincón S. Y G. Martínez (eds.). Simposio: El fitomejoramiento ante los avances científicos y tecnológicos. XIX Congreso Nacional de Fitogenética. UAAAN-SOMEFI. Buenavista, Saltillo, Coah., México.

Diaz Z., M. and M. V. Fernández C. 2009. Field performance of a liquid formulation of Azospirillum brasilense on dryland wheat productivity. Eur. J. Soil Biol. 45: 3-11.

Dobbelaere, S., A. Croonenborghs, A. Thys, D. Ptacek, J. Vanderleyden, P. Dutto, C. Labandera G., J. Caballero M., J. F. Aguirre, Y. Kapulnik, S. Brener, S. Burdman, D. Kadouri, S. Sarig, and Y. Okon. 2001. Responses of agronomically important crops to inoculation with Azospirillum. Aust. J. Plant Physiol. 28: 871-879.

Espinosa C., A., M. Tadeo R. y A. Turrent F. 2010. Concentración de la oferta de semillas mejoradas de maíz. In: C. Lira Saade. La Jornada del campo, número 30, suplemento informativo de la Jornada. México, D. F.

García O., J. G., A. Mendoza H. y N. Mayek P. 2012. Efecto de Azospirillum brasilense en el rendimiento del maíz en el norte de Tamaulipas, México. Univ. Cienc. 28: 79-84.

Gil M., A., P. A. López, O. A. Muñoz y H. López S. 2004. Variedades criollas de maíz (Zea mayz L.) en el estado de Puebla. México: Diversidad y utilización. pp. 18-25. In: J. L. Chávez S., J. Tuxill y D. I. Jarvis (eds.). Manejo de la diversidad de los cultivos en los agroecosistemas tradicionales. Instituto Internacional de Recursos Fitogenéticos. Cali, Colombia.

González, H. A. y M. A. Larios G. 1993. Estudio comparativo del rendimiento de grano en maíz: 7 híbridos experimentales, 2 variedades sintéticas y 7 criollos sobresalientes. Rev. Cienc. Agríc. Infor. 1: 19-26.

Helman Y, S. Burdman, and Y. Okon. 2011. Plant growth promotion by rhizosphere bacteria through direct effects. pp. 89-103. In: E. Rosenberg and U. Gophna (eds.). Beneficial microorganisms in multicellular life form. Springer-Verlag. Berlin, Germany.

Hungria, M., R. J. Campo, E. M. Souza, and F. A. Pedrosa. 2010. Inoculation with selected strains of Azospirillum brasilense and A. lipoferum improves yields of maize and wheat in Brazil. Plant Soil 331: 413-425.

INEGI (Instituto Nacional de Estadística y Geografía e informática). 2007. Anuario estadístico de Puebla. Tomo II. INEGI-Gobierno del Estado Puebla. INEGI. México.

Irizar G., M.B., P. Vargas V., D. Garza G., C. Tut y Couoh, I. Rojas M., A. Trujillo C., R. García S., D. Aguirre M., J. C. Martínez González, S. Alvarado Mendoza, O. Grageda Cabrera, J. Valero Garza y J. F. Aguirre Medina. 2003. Respuesta de cultivos agrícolas a los biofertilizantes en la región central de México. Agric. Téc. Méx. 29: 213-225.

Jungenheimer, R. W. 1990. Maíz variedades mejoradas, métodos de cultivo y producción de semilla. Limusa. México D.F. 
Kimetu, J. M, D. N. Mugendi, C. A.Palm, P. K. Mutuo, C. N. Gachengo, A. Bationo, S. Nandwa, and J. B. Kungu. 2004. Nitrogen fertilizer equivalencies of organics of differing quality and optimum combination with inorganic nitrogen source in Central Kenya. Nutr. Cycl. Agroecosyst. 68: 127-135.

Lafitte, H. R. and G. O. Edmeades. 1991. Three cycles of recurrent selection in maize for performance under low $\mathrm{N}$ conditions. Agron. Abst. 1991:102.

Márquez S., F., L. Sahagún, C., J. A. Carrera V. y E. Barrera G. 2000. Retrocruza limitada para el mejoramiento genético de maíces criollos. Universidad Autónoma Chapingo. Chapingo, México.

Martínez Garza, A. 1988. Diseños experimentales. Métodos y elementos y teoría. Trillas. México, D. F.

Matheus L., J. 2004. Evaluación agronómica del uso de compost de residuos de la industria azucarera (biofertilizante) en el cultivo de maíz (Zea mayz L.). Bioagro 16: 219-224.

Morales I., M. 2007 Los biofertilizantes. Una alternativa productiva, económica y sustentable. Rev. Estud. Agra. 36: 93-119.

Niño C. V., C. Nicolás M., D. J. Pérez L. y A. González H. 1998. Estudio de trece híbridos y cinco variedades de maíz en tres localidades del valle de Toluca-Atlacomulco. Rev. Cienc. Agríc. Infor. 12: 33-43.
Polanco, A. y T. Flores M. 2008. Bases para la política de I\&D e innovación de la cadena de valor del maíz. Versión 9. Foro Consultivo Científico y Tecnológico. http://foroconsultivo. org.mx/documentos/maiz. Méxcio, D. F.

SAS. Institute Inc. 2003. SAS User's GuideStatistics. Release 9.1. SAS Institute, Inc. USA.

SIAP (Servicio de Información Agroalimentaria y Pesquera). 2016. Atlas SIAP 2016. http://www.siap.gob.mx/ (Consulta: septirmbre 10, 2016).

SNIIM (Sistema Nacional de Información e Integración de Mercados) 2010. Precios de Productos Agrícolas Pecuarios y Pesqueros. http://www.economia-sniim.gob.mx/ (Consulta: diciembre 5, 2010).

Uribe, G., J. Petit y E. R. Dzib. 2007. Respuesta del cultivo de maíz a la aplicación de biofertilizantes en el sistema roza-tumba y quema en suelo alfisol (Chac-lu'um nomenclatura Maya) en Yucatán, México. Agric. Andina 13: 3-18

Whalen, J. K., Q. Hu, and A. Liu. 2003. Compost applications increase water-stable aggregates in conventional and no-tillage systems. Soil Sci. Soc. Am. J. 67: 1842-1847.

Wiersma, D. W., E. S. Oplinger, and S. O. Guy. 1986. Environment and cultivar effects on winter wheat response to ethephon plant growth regulator. Agon J. 78: 761-764. 